\title{
The smoker's paradox after successful fibrinolysis: reduced risk of reocclusion but no improved long-term cardiac outcome
}

\author{
Peter C. Kievit · Marc A. Brouwer • \\ Gerrit Veen - Wim R. M. Aengevaeren • \\ Freek W. A. Verheugt
}

Published online: 26 June 2008

(C) The Author(s) 2008

\begin{abstract}
Background In smokers treated with fibrinolysis for ST-elevation myocardial infarction (STEMI) a paradoxical beneficial short-term outcome has been reported. This was attributed to favorable clinical and angiographic baseline variables and a better response to fibrinolysis. During follow-up infarct artery reocclusion is an important prognosticator. We studied the effects of smoking on reocclusion and long-term cardiac outcome after successful fibrinolysis. Methods In the Antithrombotics in the Prevention of Reocclusion In COronary Thrombolysis trials (APRICOT-1 and -2) 499 STEMI patients with an open infarct artery $<48 \mathrm{~h}$ after fibrinolysis received randomized antithrombotic treatment until 3month follow-up angiography. Five-year clinical follow-up was complete. Results Current smokers (317 patients, 64\%) had favorable clinical (age 54 vs. 60 years, $P<0.01$ ) and angiographic (single vessel disease $61 \%$ vs. $49 \%$, $P=0.02$ ) baseline characteristics. Reocclusion rates were $21 \%(67 / 317)$ in smokers versus $32 \%$ (59/182) in nonsmokers $(P<0.01)$. Five-year infarct-free cardiac survival did not differ: $82 \%$ vs. $85 \%$. Reocclusion (HR $2.41,95 \%$ CI 1.05-5.56, $P=0.04$ ) independently predicted cardiac mortality. Smoking was independently associated with a reduced risk of reocclusion (OR 0.58 , 95\% CI $0.37-0.91$, $P=0.02$ ), but not with improved 5-year cardiac outcome (HR 1.34, 95\%CI 0.79-2.25, $P=\mathrm{ns}$ ). Conclusions After
\end{abstract}

P. C. Kievit $(\bowtie)$ - M. A. Brouwer · W. R. M. Aengevaeren · F. W. A. Verheugt

Heartlungcenter, 670 Department of Cardiology, Radboud University Nijmegen Medical Center, P.O. Box 9101, 6500 HB Nijmegen, The Netherlands

e-mail: p.kievit@cardio.umcn.nl

G. Veen

VU University Medical Center, Amsterdam, The Netherlands successful fibrinolysis, smoking is independently associated with a more than $40 \%$ reduced risk of reocclusion, which is an independent predictor of adverse outcome. However, even with more favorable baseline characteristics smokers did not have improved 5-year cardiac outcome in this low-risk population.

Keywords Smoking · Fibrinolysis · Angiography · Follow-up studies

\section{Introduction}

Many epidemiological studies have shown that cigarette smoking is associated with an increased risk of myocardial infarction and death from coronary artery disease, including sudden death [1-3]. However, after acute myocardial infarction short-term clinical outcome in smokers is more favorable than in non-smokers. In patients treated with fibrinolysis for acute myocardial infarction this so-called "smoker's paradox" has been attributed to more favorable clinical and angiographic baseline characteristics in smokers [4-6]. Several studies reported higher rates of good early antegrade epicardial flow (Thrombolysis In Myocardial Infarction (TIMI) 3 flow) in smokers [7], suggesting a better response to fibrinolysis [5, 6, 8-11].

After successful fibrinolysis, outcome is strongly related to reocclusion of the infarct artery, which portends an increased risk of death, reinfarction and revascularization [12-14]. Notably, most reocclusions occur without clinical reinfarction [12-14]. Even in these cases, reocclusion has adverse impact on recovery of left ventricular function [15]. Previously we have shown that reocclusion is a strong predictor of 10-year cardiac mortality, independent of left 
ventricular ejection fraction, and even when presenting without signs of reinfarction or recurrent ischemia [16].

Data on the risk of reocclusion in smokers are scarce and conflicting. In GUSTO-1 current smokers had a lower risk of in-hospital reocclusion than non-smokers [5], whereas no difference was observed in the TAMI trials [6].

In the angiographic follow-up studies APRICOT-1 and APRICOT-2 the effect of different antithrombotic regimens on 3-month reocclusion was studied in patients with a patent infarct artery after fibrinolysis [17, 18]. For the present analysis we assessed differences in clinical and angiographic baseline variables between smokers and nonsmokers and the effect of smoking on reocclusion and short- and long-term cardiac outcome.

\section{Methods}

Protocol

The study protocols of the APRICOT-1 (1987-1991) and APRICOT-2 (1995-2000) trials have been reported in detail previously $[17,18]$.

Patients received fibrinolysis for suspected acute STelevation myocardial infarction (STEMI) followed by infusion of unfractionated heparin. In the case of clinical and/or electrocardiographic signs of reperfusion, stable patients were asked informed consent to undergo coronary angiography within $48 \mathrm{~h}$ after fibrinolysis. Patients with a patent infarct artery were eligible for the study.

In the APRICOT-1 trial patients were randomly allocated to one of three arms: blinded treatment with either $325 \mathrm{mg}$ of aspirin or placebo, or open-label coumadin including continued heparinization until the target INR (2.8-4.0) was reached. After follow-up angiography the study medication was discontinued and, unless they had an indication for oral anticoagulation, all patients received aspirin based on the ISIS-2 results [19], which became available shortly after initiation of the trial.

In APRICOT-2 patients were randomized to two arms. In one arm patients continued treatment with aspirin $80 \mathrm{mg}$ with discontinuation of heparin at $48 \mathrm{~h}$. In the other arm coumadin was added to aspirin $80 \mathrm{mg}$ with continuation of heparin until moderate-intensity anticoagulation (target INR 2-3) was achieved.

\section{Cardiac catheterization}

Follow-up angiography was scheduled at 3 months to assess reocclusion, the primary endpoint of the APRICOT trials. In APRICOT-1 reocclusion was defined as ECSG grade 4 (91-99\% diameter stenosis, no complete filling within 3 cycles) or grade 5 stenosis (total occlusion with or without collateral filling) [17]. In APRICOT-2 the definition of reocclusion was TIMI flow grade 2 or less [18]. Anatomical reocclusions were defined as ECSG grade 5 and TIMI flow 0 or 1 , respectively. An ischemia-guided revascularization strategy was adopted [17]. If coronary angioplasty was performed before the planned repeat angiography, the patency status of the infarct artery before wire crossing was scored as follow-up endpoint. At baseline angiography quantitative coronary angiography (QCA) of the culprit lesion was performed, providing a precise estimation of the diameter stenosis with low intra- and interobserver variability [20]. For this analysis the optimal single projection with the most severe measured stenosis was used. Morphology of the culprit lesion was scored according to the Ambrose criteria [21].

\section{Clinical follow-up}

Five-year clinical and survival data were collected from medical charts, civil registries, or by written or telephone contact with the patient, relatives or the general practitioner. All deaths were confirmed by civil registry data. Patients participating in APRICOT-2 were systematically questioned about their smoking behavior at follow-up visits. Cardiac mortality was defined based on the information of the treating physician, and only in the case of a confirmed cardiac diagnosis. An instantaneous death, for which the cause could not be determined with certainty, was scored as a death of cardiac origin. Confirmed vascular deaths (strokes, aneurysms, aortic dissections, pulmonary embolisms) were not considered as cardiac death. If the cause was unknown/uncertain and death did not occur instantaneously it was classified as 'cause undetermined'. An event was qualified as reinfarction (infarct artery related) when at least 3 of the 4 GUSTO- 1 criteria were met [22]: (1) recurrent ischemic symptoms lasting $>15 \mathrm{~min}$ after resolution of symptoms of the index infarction, (2) new ST-T wave changes or new Q-waves in the same area as the index infarction, (3) a second elevation in cardiac enzymes over the normal upper limit (or by a further $20 \%$ if already elevated), and (4) reocclusion of the infarct artery. Reinfarctions not meeting these electrocardiographic criteria or attributed to a different coronary artery at urgent repeat angiography were considered to be not infarct artery related.

Recurrent ischemic events were defined as the combination of reinfarction, unstable angina and recurrent angina with documented ischemia at stress testing. Revascularization of the infarct artery was scored in the case of percutaneous coronary intervention (PCI) of the index infarct vessel or coronary artery bypass grafting (CABG). 
Statistics

Statistical analysis was performed with SPSS 14.0 (SPSS Inc. 2005, Chicago, USA). For comparison of continuous variables the Student's $t$-test and Mann-Whitney $U$-test were used whenever appropriate. Comparisons of proportions between groups were performed with the $\chi^{2}$-test. Statistical significance was defined as a two-sided $P$-value $<0.05$. Multivariable logistic regression analysis was used to determine the association between smoking status at baseline and reocclusion. Variables included in the model were baseline characteristics that were imbalanced between smokers and non-smokers $(P<0.10$, see "Results"), predictors of reocclusion at univariable analysis and the antithrombotic regimen (prespecified).

Long-term survival analysis was performed according to Kaplan and Meier, using the log rank test for comparison between groups. To determine the association between smoking status at baseline and 5-year (infarct-free) cardiac survival multivariable Cox-regression analysis using forward logistic regression was performed. TIMI risk score variables [23] and the antithrombotic regimen were prospectively chosen to be included in the model. Baseline characteristics that were imbalanced between smokers and non-smokers and univariate predictors of long-term outcome completed the model.

\section{Results}

Of the 558 patients enrolled in the APRICOT-1 and APRICOT-2 trials the current report includes the 499 patients with complete angiographic follow-up (Fig. 1). Patients without follow-up angiography were significantly older (62 \pm 8 vs. $56 \pm 10$ years, $P<0.01$ ), more often female (33\% vs. $17 \%$, $P<0.01)$, and tended to have more often a history of previous myocardial infarction ( $16 \%$ vs. $9 \%, P=0.09$ ). The proportion of current smokers at enrollment did not differ from patients with angiographic follow-up (59\% vs. $64 \%, P=\mathrm{ns})$.

Baseline characteristics

At baseline 317 (64\%) of the 499 patients were current smokers. Baseline characteristics of smokers and nonsmokers are listed in Table 1 . With respect to clinical characteristics smokers were younger, and had less often a history of prior myocardial infarction, diabetes, hypertension or a positive family history.

At baseline angiography smokers had less severe residual infarct related stenoses, more smooth infarct lesions and more often single vessel disease. Smokers tended to have more often an infarction related to the left coronary artery. Peak CK-levels were higher in smokers, but baseline ejection fraction did not differ from non-smokers.
Fig. 1 Flow chart showing number of patients from APRICOT-1 and APRICOT-2 excluded and number remaining with follow-up angiography and long-term clinical follow-up

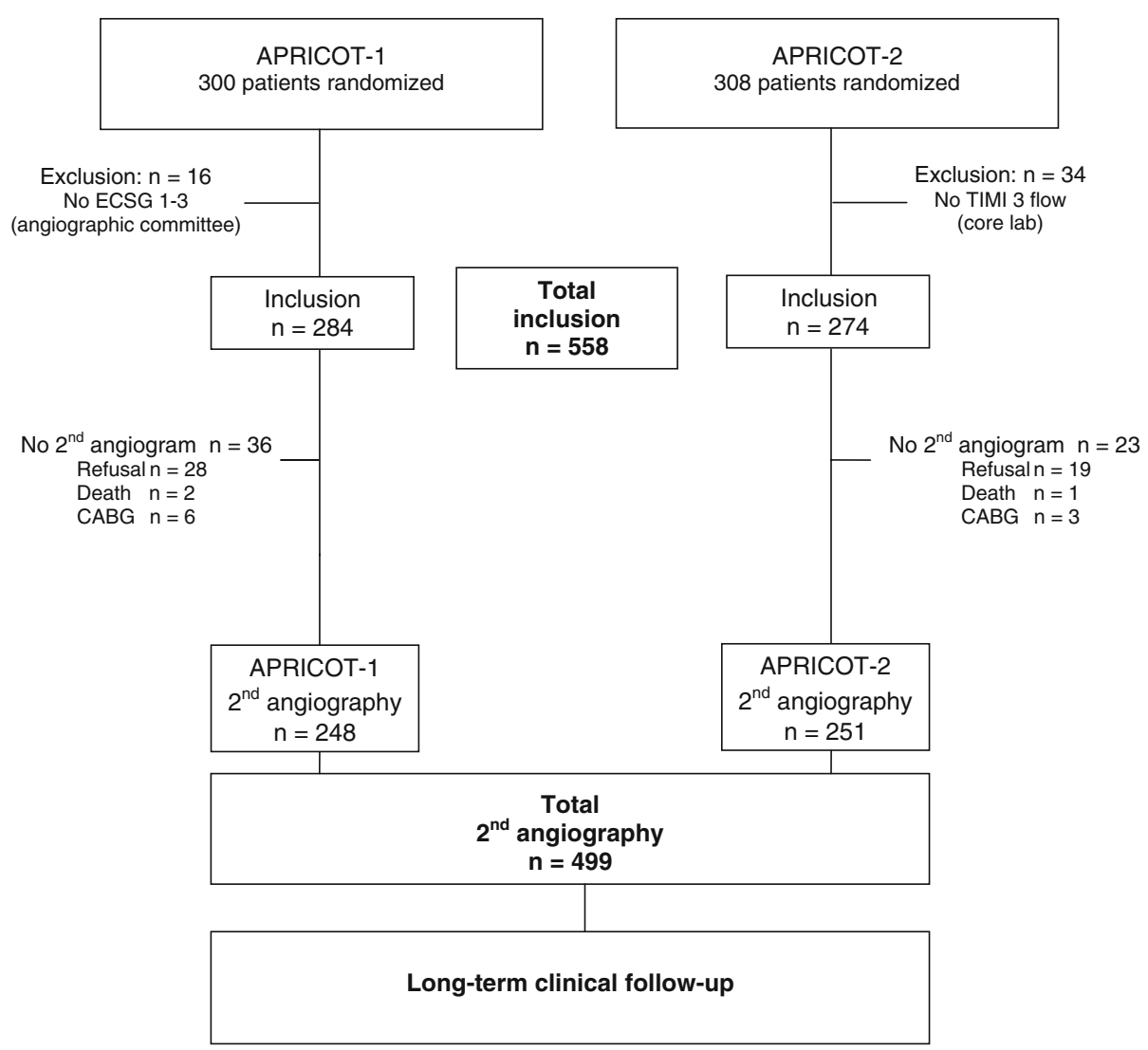


Table 1 Baseline characteristics

\begin{tabular}{|c|c|c|c|}
\hline & $\begin{array}{l}\text { Smokers } \\
(\mathrm{n}=317)\end{array}$ & $\begin{array}{l}\text { Non-smokers } \\
(\mathrm{n}=182)\end{array}$ & $P$ \\
\hline \multicolumn{4}{|l|}{ Clinical characteristics } \\
\hline Men & $260(82)$ & $154(85)$ & 0.46 \\
\hline Age (years) & $54 \pm 9$ & $60 \pm 9$ & $<0.01$ \\
\hline Previous angina & $161(51)$ & $106(58)$ & 0.28 \\
\hline $\begin{array}{l}\text { Previous myocardial } \\
\text { infarction }\end{array}$ & $18(6)$ & $25(14)$ & $<0.01$ \\
\hline Diabetes & $11(4)$ & $15(8)$ & 0.02 \\
\hline Hypertension & $68(22)$ & $57(31)$ & 0.02 \\
\hline Family history of CAD & $107(34)$ & $85(47)$ & $<0.02$ \\
\hline Hypercholesterolemia & $213(67)$ & $123(68)$ & 0.93 \\
\hline $\begin{array}{l}\text { Time symptoms- } \\
\text { fibrinolysis (h) }\end{array}$ & $2.2 \pm 1.2$ & $2.2 \pm 1.2$ & 0.87 \\
\hline $\begin{array}{l}\text { Peak creatine kinase } \\
\text { (units/l) }\end{array}$ & $\begin{array}{l}1116 \\
\quad(563-2146)\end{array}$ & $\begin{array}{l}862 \\
\quad(401-1825)\end{array}$ & $<0.01$ \\
\hline \multicolumn{4}{|l|}{ Antithrombotic regimen } \\
\hline Coumadin & $60(19)$ & $21(12)$ & 0.06 \\
\hline Aspirin $325 \mathrm{mg}$ & $57(18)$ & $36(20)$ & \\
\hline Placebo & $52(16)$ & $22(12)$ & \\
\hline Aspirin $80 \mathrm{mg}$ & $71(22)$ & $57(31)$ & \\
\hline $\begin{array}{l}\text { Aspirin } 80 \mathrm{mg} \\
+ \text { Coumadin }\end{array}$ & $77(24)$ & $46(25)$ & \\
\hline \multicolumn{4}{|c|}{ Angiographic characteristics } \\
\hline \multicolumn{4}{|l|}{ Infarct artery } \\
\hline LAD & $138(44)$ & $68(37)$ & 0.06 \\
\hline LCX & $54(17)$ & $23(13)$ & \\
\hline $\mathrm{RCA}$ & $125(39)$ & $91(50)$ & \\
\hline $\begin{array}{l}\text { Culprit stenosis severity } \\
(\%, \text { QCA })\end{array}$ & $58.8 \pm 14$ & $61.6 \pm 12$ & 0.02 \\
\hline \multicolumn{4}{|l|}{ Culprit lesion morphology } \\
\hline Smooth & $204(64)$ & $94(52)$ & $<0.01$ \\
\hline Complex & $113(36)$ & $88(48)$ & \\
\hline Single vessel disease & $192(61)$ & $90(49)$ & 0.02 \\
\hline $\begin{array}{l}\text { Left ventricular ejection } \\
\text { fraction }(\%)\end{array}$ & $52.4 \pm 11$ & $53.5 \pm 10$ & 0.29 \\
\hline
\end{tabular}

Data are presented as number $(\%)$ of subjects for discrete variables and mean \pm standard deviation for continuous variables, except for peak creatine kinase which is presented as median (interquartile range). $\mathrm{CAD}=$ coronary artery disease $\mathrm{LAD}=$ left anterior descending coronary artery; $\mathrm{LCX}=$ left circumflex coronary artery; RCA = right coronary artery; $\mathrm{QCA}=$ quantitative coronary angiography

\section{Reocclusion}

Follow-up angiography was performed after a median of 89 (interquartile range 64-97) days. Reocclusion rates were $21 \%$ (67/317) in smokers versus $32 \%$ (59/182) in nonsmokers: RR $0.56,95 \%$ CI $0.37-0.84, P<0.01$. When only anatomical reocclusions were considered the rates were $16 \%$ (49/317) and 25\% (45/182), respectively: RR 0.56, 95\%CI $0.35-0.88, P=0.01$.
Exploratory analysis showed that the reduced risk of reocclusion observed in smokers in the overall study population was generally consistent across many different subgroups (Fig. 2). No significant interaction $(P<0.01)$ between the effect of smoking and any of the subgroup variables was observed.

At multivariable analysis smoking was independently associated with a markedly reduced risk of reocclusion of the infarct artery: OR $0.58,95 \%$ CI $0.37-0.91, P=0.02$. If only anatomical reocclusions (ECSG grade 5, TIMI 0,1) were considered the adjusted odds ratio was $0.59,95 \% \mathrm{CI}$ $0.35-0.97, P=0.04$. Baseline culprit stenosis severity also independently predicted reocclusion: OR $1.07,95 \% \mathrm{CI}$ 1.05-1.09, $P<0.01$.

Compared with placebo, antithrombotic treatment with aspirin alone $(325 \mathrm{mg}$ or $80 \mathrm{mg}, \mathrm{n}=221$; OR $0.51,95 \% \mathrm{CI}$ $0.27-0.98, P=0.04)$ or in combination with coumadin (n $=123$; OR 0.29 , 95\%CI $0.13-0.65, P<0.01$ ) was independently associated with a reduced risk of reocclusion. Coumadin alone $(\mathrm{n}=81$; OR 0.71 , 95\%CI $0.33-$ $1.53, P=$ ns) was not superior to placebo.

Clinical outcome-short-term

Table 2 lists the 3-month rates of recurrent ischemic events and revascularizations in smokers and non-smokers. Reinfarction rates were similar in smokers and nonsmokers. Smokers had lower rates of unstable angina and revascularization. The proportion of patients with recurrent ischemic events who underwent revascularization was $43 \%$ (39/90) in smokers and 49\% (37/75) in non-smokers $(P=\mathrm{ns})$.

\section{Clinical presentation of reocclusion}

Reocclusion presented with reinfarction in $21 \%$ (26/126) of cases. The proportion of reocclusions presenting with reinfarction did not differ between smokers and nonsmokers: $21 \%(14 / 67)$ vs. $20 \%$ (12/59), $P=$ ns. In both groups the proportion of reocclusions occurring with recurrent ischemic events was also similar: $51 \%$ (34/67) vs. $58 \%(34 / 59), P=$ ns.

Of the patients with reocclusion $27 \%$ (34/126) underwent revascularization at the time of follow-up angiography with no difference between groups: $25 \%$ (17/67) in smokers versus $29 \%(17 / 59)$ in non-smokers $(P=\mathrm{ns})$.

Clinical outcome-long-term

Data on 5-year survival were complete in all 499 patients (100\%). The long-term use of antithrombotic agents (99\%), beta-blockers (70\%), ACE-inhibitors (45\%) and statins (70\%) did not differ between smokers and non-smokers. 
Fig. 2 Subgroup analyses on the odds of reocclusion in smokers and non-smokers after successful fibrinolysis

\begin{tabular}{|c|c|}
\hline Variable & Odds ratio $(95 \% \mathrm{Cl}$ \\
\hline Overall & $0.56(0.37-0.84)$ \\
\hline \multicolumn{2}{|l|}{ Sex } \\
\hline Male & $0.61(0.39-0.95)$ \\
\hline Female & $0.35(0.12-1.03)$ \\
\hline \multicolumn{2}{|r|}{ (10) } \\
\hline$\geq 65 \mathrm{yr}$ & $1.28(0.54-3.04)$ \\
\hline$<65 \mathrm{yr}$ & $0.47(0.29-0.76)$ \\
\hline \multicolumn{2}{|l|}{ Previous MI } \\
\hline yes & $0.26(0.06-1.11)$ \\
\hline no & $0.62(0.40-0.96)$ \\
\hline \multicolumn{2}{|l|}{ Diabetes } \\
\hline yes & $1.14(0.22-5.84)$ \\
\hline no & $0.54(0.34-0.87)$ \\
\hline \multicolumn{2}{|l|}{ Hypertension } \\
\hline yes & $0.45(0.19-1.07)$ \\
\hline no & $0.57(0.36-0.92)$ \\
\hline \multicolumn{2}{|l|}{ Family history of CAD } \\
\hline yes & $0.59(0.30-1.13)$ \\
\hline no & $0.53(0.31-0.90)$ \\
\hline \multicolumn{2}{|l|}{ Cholesterol } \\
\hline$\geq 5.0 \mathrm{mmol} / \mathrm{L}$ & $0.61(0.38-0.99)$ \\
\hline$<5.0 \mathrm{mmol} / \mathrm{L}$ & $0.42(0.18-0.96)$ \\
\hline \multicolumn{2}{|l|}{ Infarct artery } \\
\hline LAD & $0.66(0.35-1.25)$ \\
\hline LCX & $0.22(0.07-0.65)$ \\
\hline $\mathrm{RCA}$ & $0.63(0.33-1.18)$ \\
\hline \multicolumn{2}{|l|}{ Culprit stenosis severity (QCA) } \\
\hline$\geq 50 \%$ & $0.60(0.38-0.94)$ \\
\hline$<50 \%$ & $0.34(0.08-1.37)$ \\
\hline \multicolumn{2}{|l|}{ Culprit lesion morphology } \\
\hline smooth & $0.51(0.30-0.86)$ \\
\hline complex & $0.57(0.29-1.13)$ \\
\hline \multicolumn{2}{|l|}{ Extent of CAD } \\
\hline single vessel disease & $0.55(0.31-0.97)$ \\
\hline multi vessel disease & $0.59(0.33-1.08)$ \\
\hline \multicolumn{2}{|l|}{ Ejection fraction } \\
\hline$\geq 50 \%$ & $0.56(0.33-0.93)$ \\
\hline & $0.53(0.26-1.09)$ \\
\hline \multicolumn{2}{|l|}{ Antithrombotic regimen } \\
\hline Coumadin & $0.19(0.06-0.55)$ \\
\hline Aspirin $325 \mathrm{mg}$ & $0.61(0.23-1.57)$ \\
\hline Placebo & $0.44(0.16-1.25)$ \\
\hline Aspirin $80 \mathrm{mg}$ & $0.86(0.40-1.86)$ \\
\hline Aspirin $80 \mathrm{mg}+$ Coumadin & $0.61(0.23-1.65)$ \\
\hline
\end{tabular}

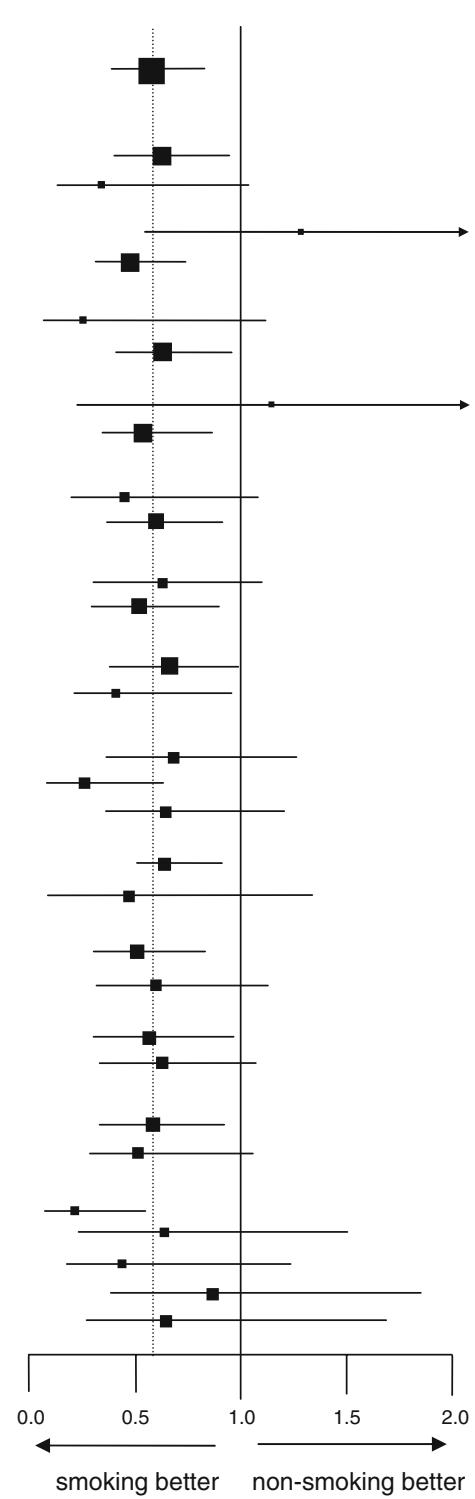

$\mathrm{MI}=$ myocardial infarction; $\mathrm{CAD}=$ coronary artery disease; LAD = left anterior descending coronary artery; LCX = left circumflex coronary artery; RCA = right coronary artery: $\mathrm{QCA}$ = quantitative coronary angiography
Table 2 Three-month clinical outcome

\begin{tabular}{lllr}
\hline & $\begin{array}{l}\text { Smokers } \\
(\mathrm{n}=317)\end{array}$ & $\begin{array}{l}\text { Non-smokers } \\
(\mathrm{n}=182)\end{array}$ & $P$ \\
\hline Recurrent ischemic events $^{\text {Reinfarction }}{ }^{\mathrm{a}}$ & $90(28)$ & $75(41)$ & $<0.01$ \\
$\quad 20(6)$ & $14(8)$ & 0.56 \\
$\quad \begin{array}{l}\text { Unstable angina } \\
\begin{array}{l}\text { Recurrent angina with } \\
\text { documented ischemia }\end{array}\end{array}$ & $49(16)$ & $46(25)$ & $<0.01$ \\
$\begin{array}{l}\text { Revascularization } \\
\text { a }\end{array}$ & $43(14)$ & $15(8)$ & 0.50 \\
PCI & $41(13)$ & $41(23)$ & 0.01 \\
CABG & $5(2)$ & $4(2)$ & $<0.01$ \\
\hline
\end{tabular}

Data are presented as number of subjects and proportions $(\%)$ per study group. Patients may have had events in more than one category

a Infarct artery related

${ }^{\mathrm{b}}$ Positive stress test
During follow-up 38 deaths were observed, 29 (76\%) of which were cardiac. Five-year survival was $93 \%$ in smokers and $92 \%$ in non-smokers $(P=$ ns). Cardiac survival did not differ either: 94 and 94\%, respectively. Cardiac survival free from infarct artery related reinfarction was $82 \%$ in smokers and $85 \%$ in non-smokers (Fig. 3, $P=$ ns). Including revascularizations event-free 5-year survival did not differ either: 58 and $54 \%$, respectively $(P=\mathrm{ns})$.

At multivariable Cox-regression analysis reocclusion (HR 2.41, 95\%CI 1.05-5.56, $P=0.04$ ) and left ventricular ejection fraction (HR 0.93, 95\%CI 0.90-0.97, $P<0.01$ ) independently predicted 5-year cardiac mortality. Smoking was neither predictive of long-term cardiac survival (HR $1.42,95 \% \mathrm{CI} 0.55-3.63, P=\mathrm{ns})$ nor of infarct-free cardiac survival (HR 1.34, 95\%CI 0.79-2.25, $P=\mathrm{ns}$ ). 


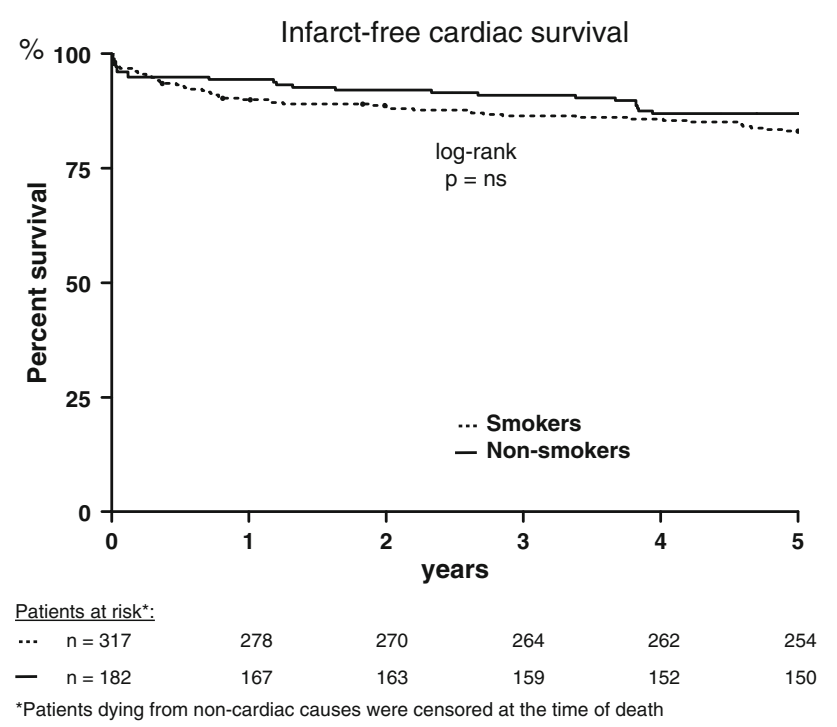

Fig. 3 Estimated cardiac survival free from infarct artery related reinfarction in smokers and non-smokers after successful fibrinolysis

\section{Discussion}

This is one of the largest reports on the impact of smoking on reocclusion in patients with a patent infarct artery after fibrinolysis for STEMI. Smoking was independently associated with a markedly reduced risk of reocclusion at 3 months. However, even with more favorable clinical and angiographic baseline characteristics, smokers did not have improved 5-year cardiac outcome.

Baseline characteristics of smokers after successful fibrinolysis

In previous studies after fibrinolysis the paradoxical beneficial short-term outcome of smokers has been attributed to more favorable clinical and angiographic baseline characteristics and a better response to fibrinolytic therapy [4-6, 8-11]. Consistent with the latter observation we found a rate of current smokers in patients after successful fibrinolysis which is considerably higher than reported in the overall fibrinolysis population in contemporary megatrials: $64 \%$ vs. $44 \%[22,24]$. Also in our study smokers had a more favorable clinical risk profile with younger age and less concomitant risk factors for coronary artery disease. At baseline angiography smokers had less extensive coronary artery disease with a higher rate of single vessel disease, less severe residual infarct related stenoses, and more smooth infarct lesions.

Whereas in previous trials smokers most often had infarctions related to the right coronary artery $[4,5,8]$, left coronary artery infarctions predominated in smokers in our study. Besides a different timing of angiography (24 h vs. $90 \mathrm{~min}$ ), this may be explained by the inclusion of only patients with a patent infarct artery. Smoking status predicts TIMI 3 flow independent of the infarct artery [11].

Smoking and reocclusion

Given the strong relationship between reocclusion and outcome after successful fibrinolysis [12-14], previous studies addressed the risk of reocclusion in smokers in search for potential mechanisms explaining their favorable outcome. Results of these studies have been conflicting. In GUSTO-1 5-7 days follow-up angiography was performed in $75 \%$ of patients with a patent infarct artery (TIMI 2,3) at 90-min angiography who were randomized to undergo a second angiogram [25]. The unadjusted reocclusion rate (TIMI 0,1) was significantly lower in current smokers than in non-smokers: $2.7 \%$ vs. $7.7 \%$ [5]. However, in the TAMI trials predischarge reocclusion rates in the subgroup of patients who did not undergo acute angioplasty were similar in smokers and non-smokers: $7.3 \%$ vs. 9.3\% [6]. After primary angioplasty (half of patients receiving stents) crude 7 -month reocclusion rates did not differ either: $7.7 \%$ vs. $9.3 \%$ [26].

The APRICOT-trials were specifically designed for the study of reocclusion in patients with a patent infarct artery after fibrinolysis and included mandatory follow-up angiography, which was performed in $89 \%$ of patients (Fig. 1). Whereas previous studies only reported unadjusted reocclusion rates, we found a more than $40 \%$ reduced risk of reocclusion in smokers, even after multivariable analysis. The reduced risk was observed consistently across many different subgroups and occurred independent of antithrombotic regimen and culprit stenosis severity, a known strong predictor of reocclusion in this setting [27-29]. The majority of reocclusions have been shown to occur without clinical reinfarction [12-14]. In our study reocclusion occurred without clinical reinfarction in almost $80 \%$ of cases, while $46 \%$ of patients with reocclusion did not experience recurrent ischemic events. The clinical presentation of reocclusion did not differ between smokers and non-smokers.

The mechanism behind the reduced risk of reocclusion in smokers may be related to the nature of coronary occlusions in smokers. Coronary lesions in smokers, with their younger age and less extensive coronary artery disease, are presumably more thrombotic than atherogenic in nature [6]. Thrombotic lesions are more susceptible to regression of the culprit stenosis, which has been shown to occur before hospital discharge in patients treated with fibrinolysis [30]. Whereas stenosis severity between smokers and non-smokers did not differ in studies with 90min angiography [5, 6, 9, 10], a lower diameter stenosis has been reported in smokers before discharge [6]. In our study with 24-h angiography smokers also had a lower culprit 
diameter stenosis. These findings suggest that regression of the culprit stenosis in the first days after fibrinolysis may be more pronounced in smokers than in non-smokers. Resolution of thrombus material on a primarily non-atherogenic lesion may reduce the residual culprit stenosis and thereby the risk of reocclusion.

\section{Smoking and clinical outcome}

A lower risk of short-term mortality in smokers has been reported in a broad range of patients after acute myocardial infarction: after fibrinolysis [4-6, 9, 10, 31] after primary angioplasty [26] and in patients with heart failure or left ventricular dysfunction [32]. In most [4-6, 9, 10, 26], but not all [31] of these reports, the survival benefit was attributed to the favorable clinical and angiographic baseline characteristics in smokers. In our study smokers also had a more favorable clinical and angiographic risk profile. Moreover, smokers had a more than $40 \%$ reduced risk of reocclusion. As previously observed with respect to 10-year cardiac outcome in APRICOT-1 [16], also in the present study reocclusion was independently associated with a more than twofold increased risk of long-term cardiac mortality. Nevertheless, no survival difference was found between smokers and non-smokers. Notably, both in smokers and non-smokers most patients had preserved left ventricular function (mean ejection fraction $>50 \%$ ). This may explain the low 5-year cardiac mortality rate (about 6\%) in our study and also that, as a consequence, the moderate absolute difference in risk of reocclusion (11\%) was not large enough to yield a difference in survival between smokers and nonsmokers. Our findings resemble observations from GUSTO $\mathrm{V}$ [24], in which study a significant reduction in reinfarction did not result in improved short-term survival, despite the strong independent relationship between reinfarction and mortality after fibrinolysis [33]. Analogous to that finding, a reduced risk of reocclusion may not automatically confer improved outcome, especially in a low-risk population.

Besides the adverse impact on long-term survival, reocclusion also impairs left ventricular recovery [15]. The lower reocclusion rate in smokers may therefore confer a beneficial effect on remodeling and a lower risk to develop heart failure. Although we did not assess patients' functional status, admissions for heart failure were infrequent and did not differ between groups.

Several studies have reported lower reinfarction rates in smokers as compared to non-smokers [4, 5, 26, 31, 32]. In one study current smoking was independently associated with a lower risk of reinfarction 42 days after fibrinolysis [4]. Despite the independently reduced risk of reocclusion in smokers in our study, the risk of reinfarction at 3 months did not differ from non-smokers. The proportion of reocclusions presenting with reinfarction was only about $20 \%$, and did not differ between smokers and non-smokers. With the $11 \%$ absolute difference in reocclusion rate between groups and the overall 3-month reinfarction rate of only $7 \%$ this did not result in a difference in risk of reinfarction between smokers and non-smokers. Notably, after fibrinolysis about half of the reinfarctions occur in the first $48 \mathrm{~h}$ [33]. The design of our study, with inclusion of only clinically stable patients with a patent infarct artery at 24$48 \mathrm{~h}$, precluded detection of a difference in the rate of these very early reinfarctions.

Non-smokers had a higher rate of early recurrent ischemic events, and, as a result of the ischemia-guided revascularization strategy, more often underwent early revascularization. Whereas routine revascularization early after fibrinolysis may be preventive of recurrent events [34], ischemia-guided revascularization was not associated with improved outcome in this study.

Data on smoking cessation during follow-up were only available in patients from APRICOT-2. About half of the smokers stopped immediately at the time of the index infarction and did not resume smoking. Despite the strong association between smoking cessation after acute myocardial infarction and improved outcome in past trials [35, 36], 5-year infarct free survival in these "quitters" did not differ from patients who continued to smoke or nonsmokers (data not shown).

\section{Limitations}

Our observations apply to a well defined, but selected group of low-risk patients. Although this is a post hoc pooled analysis from two trials, the study protocols were almost identical and the reduced risk of reocclusion in smokers was present in both trials and consistent across many different subgroups.

The definition of reocclusion differed between APRICOT1 and APRICOT-2. However, if only anatomical reocclusions (ECSG grade 5 and TIMI 0,1) were considered the observed effect of smoking on reocclusion did not change.

Five-year survival data were complete in all patients and all deaths were confirmed by civil registry data. Despite strenuous efforts to obtain detailed information about the circumstances of death in all deceased patients and a strict definition of cardiac mortality, the adjudication of mortality may sometimes have been limited by the recollection of patients' relatives and general practitioners. In addition, we cannot exclude that non-fatal events may not have been reported.

The fact that APRICOT-2 was performed a decade later than APRICOT-1, in an era in which the use of ACEinhibitors and statins became widespread, does not seem to have affected our results. To date treatment of patients after fibrinolysis is more aggressive than in the APRICOT trials, 
with higher rates of revascularization and improved antithrombotic therapy (new anticoagulants, clopidogrel). In the current era the risk of reocclusion and outcome in smokers after fibrinolysis may therefore be different than observed in this study.

\section{Conclusions}

Also in patients with a patent infarct artery within $48 \mathrm{~h}$ after fibrinolysis, smokers have more favorable clinical and angiographic baseline characteristics than non-smokers. Smoking was independently associated with a more than $40 \%$ reduced risk of reocclusion. However, although reocclusion independently predicted cardiac survival, the markedly reduced risk of reocclusion in smokers did not lead to improved outcome in this population of patients with a low a priori risk of adverse events.

Open Access This article is distributed under the terms of the Creative Commons Attribution Noncommercial License which permits any noncommercial use, distribution, and reproduction in any medium, provided the original author(s) and source are credited.

\section{References}

1. Kannel WB (1981) Update on the role of cigarette smoking in coronary artery disease. Am Heart J 101:319-328. doi:10.1016/ 0002-8703(81)90197-6

2. Doll R, Peto R, Wheatley K, Gray R, Sutherland I (1994) Mortality in relation to smoking: 40 years' observations on male British doctors. BMJ 309:901-911

3. Teo KK, Ounpuu S, Hawken S et al (2006) Tobacco use and risk of myocardial infarction in 52 countries in the INTERHEART study: a case-control study. Lancet 368:647-658. doi:10.1016/ S0140-6736(06)69249-0

4. Mueller HS, Cohen LS, Braunwald E et al (1992) Predictors of early morbidity and mortality after thrombolytic therapy of acute myocardial infarction. Analyses of patient subgroups in the Thrombolysis in Myocardial Infarction (TIMI) trial, phase II. Circulation 85:1254-1264

5. Barbash GI, Reiner J, White HD et al (1995) Evaluation of paradoxic beneficial effects of smoking in patients receiving thrombolytic therapy for acute myocardial infarction: mechanism of the smoker's paradox from the GUSTO-1 trial with angiographic insights. J Am Coll Cardiol 26:1222-1229. doi:10.1016/ 0735-1097(95)00299-5

6. Grines CL, Topol EJ, O'Neil WW et al (1995) Effect of cigarette smoking on outcome after thrombolytic therapy for myocardial infarction. Circulation 91:298-303

7. TIMI Study Group (1985) The Thrombolysis in Myocardial Infarction (TIMI) trial. Phase I findings. N Engl J Med 312:932-936

8. Gomez MA, Karagounis LA, Allen A, Anderson JL, for the TEAM-2 investigators (1993) Effect of cigarette smoking on coronary patency after thrombolytic therapy for myocardial infarction. Am J Cardiol 72:373-378. doi:10.1016/0002-9149(93)91125-2

9. Zahger D, Cercek B, Cannon CP et al (1995) How do smokers differ from nonsmokers in their response to thrombolysis? (The TIMI-4 trial). Am J Cardiol 75:232-236. doi:10.1016/0002-9149(95) 80026-O
10. Angeja BG, Kermgard S, Chen MS et al (2002) The smoker's paradox: Insights from the angiographic substudies of the TIMI trials. J Thromb Thrombolysis 13:133-139. doi:10.1023/A:1020 470721977

11. Lundergan CF, Reiner JS, McCarthy WF et al (1998) Clinical predictors of early-infarct-related artery patency following thrombolytic therapy: importance of body weight, smoking history, infarct-related artery and choice of thrombolytic regimen: the GUSTO-1 experience. J Am Coll Cardiol 32:641-647. doi: 10.1016/S0735-1097(98)00278-2

12. Ohman EM, Califf RM, Topol EJ et al (1990) Consequences of reocclusion after successful reperfusion therapy in acute myocardial infarction. TAMI Study Group. Circulation 82: 781-791

13. Brouwer MA, Böhncke JR, Veen G et al (1995) Adverse longterm effects of reocclusion after coronary thrombolysis. J Am Coll Cardiol 26:1440-1444. doi:10.1016/0735-1097(95)00355-X

14. Verheugt FWA, Meijer A, Lagrand WK et al (1996) Reocclusion: the flip side of coronary thrombolysis. J Am Coll Cardiol 27:766773. doi:10.1016/0735-1097(95)00492-0

15. Meijer A, Verheugt FWA, van Eenige MJ, Werter CJ (1994) Left ventricular function at 3 months after successful thrombolysis. Impact of reocclusion without reinfarction on ejection fraction, regional function, and remodeling. Circulation 90:1706-1714

16. Brouwer MA, Kievit PC, Dieker HJ, Veen G, Karreman AJ, Verheugt FWA (2008) Sustained coronary patency after fibrinolytic therapy as independent predictor of 10-year cardiac survival. Observations from the APRICOT-trial. Am Heart J 155:10391046. doi:10.1016/j.ahj.2008.01.008

17. Meijer A, Verheugt FWA, Werter CJ et al (1993) Aspirin versus coumadin in the prevention of reocclusion and recurrent ischemia after successful thrombolysis: a prospective placebo-controlled angiographic study. Results of the APRICOT Study. Circulation 87:1524-1530

18. Brouwer MA, Van den Bergh PJPC, Aengevaeren WRM et al (2002) Aspirin plus medium intensity coumadin versus aspirin alone in the prevention of reocclusion after successful thrombolysis for suspected acute myocardial infarction: results of the APRICOT2 study. Circulation 106:659-665. doi:10.1161/01.CIR.00000 24408.81821 .32

19. ISIS-2 Collaborative Group (1988) Randomised trial of intravenous streptokinase, oral aspirin, both, or neither among 17, 187 cases of suspected acute myocardial infarction: ISIS-2. Lancet 2:349-360

20. Reiber JHC, van der Zwet PMJ, Koning G et al (1993) Accuracy and precision of quantitative digital coronary arteriography: observer-, short- and medium-term variabilities. Cathet Cardiovasc Diagn 28:187-198

21. Ambrose JA, Winters SL, Arora RR et al (1985) Coronary angiographic morphology in myocardial infarction: a link between the pathogenesis of unstable angina and myocardial infarction. J Am Coll Cardiol 6:1233-1238

22. GUSTO Investigators (1993) An international randomized trial comparing four thrombolytic strategies for acute myocardial infarction. N Engl J Med 329:673-682. doi:10.1056/NEJM19 9309023291001

23. Morrow DA, Antman EM, Charlesworth A et al (2000) TIMI risk score for ST-elevation myocardial infarction: a convenient, bedside, clinical score for risk assessment at presentation: an intravenous $\mathrm{nPA}$ for treatment of infarcting myocardium early II trial substudy. Circulation 102:2031-2037

24. The GUSTO V Investigators (2001) Reperfusion therapy for acute myocardial infarction with fibrinolytic therapy or combination reduced fibrinolytic therapy and platelet glycoprotein IIb/ IIIa inhibition: the GUSTO V randomised trial. Lancet 357:19051914. doi:10.1016/S0140-6736(00)05059-5 
25. The GUSTO Angiographic Investigators (1993) The effects of tissue plasminogen activator, streptokinase, or both on coronary-artery patency, ventricular function, and survival after acute myocardial infarction. N Engl J Med 329:1615-1622. doi:10.1056/NEJM1993 11253292204

26. Weisz G, Cox DA, Garcia E et al (2005) Impact of smoking status on outcomes of primary coronary intervention for acute myocardial infarction - the smoker's paradox revisited. Am Heart J 150:358-364. doi:10.1016/j.ahj.2004.01.032

27. Veen G, Meijer A, Verheugt FWA et al (1993) Culprit lesion morphology and stenosis severity in the prediction of reocclusion after coronary thrombolysis: angiographic results of the APRICOT study. Antithrombotics in the Prevention of Reocclusion In COronary Thrombolysis. J Am Coll Cardiol 22:1755-1762

28. French JK, Ellis CJ, Webber BJ et al (1998) Abnormal coronary flow in infarct arteries 1 year after myocardial infarction is predicted at 4 weeks by corrected Thrombolysis in Myocardial Infarction (TIMI) frame count and stenosis severity. Am J Cardiol 81:665-671. doi:10.1016/S0002-9149(97)01004-7

29. Kievit PC, Brouwer MA, Veen G, Karreman J, Verheugt FWA (2004) High-grade infarct related stenosis after successful thrombolysis: strong predictor of reocclusion, but not of clinical reinfarction. Am Heart J 148:826-833. doi:10.1016/j.ahj.2004. 05.043

30. Topol EJ, Califf RM, George BS et al (1987) A randomized trial of immediate versus delayed elective angioplasty after intravenous tissue plasminogen activator in acute myocardial infarction. N Engl J Med 317:581-588
31. Barbash GI, White HD, Modan M et al (1993) Significance of smoking in patients receiving thrombolytic therapy for acute myocardial infarction. Experience gleaned from the international tissue plasminogen activator/streptokinase mortality trial. Circulation 87:53-58

32. Jaatun HJ, Sutradhar SC, Dickstein K (2004) Comparison of mortality rates after acute myocardial infarction in smokers versus non-smokers. Am J Cardiol 94:632-636. doi:10.1016/j.amjcard. 2004.05.029

33. Gibson CM, Karha J, Murphy SA et al (2003) Early and longterm clinical outcomes associated with reinfarction following fibrinolytic administration in the Thrombolysis in Myocardial Infarction trials. J Am Coll Cardiol 42:7-16. doi:10.1016/S07351097(03)00506-0

34. Collet JP, Montalescot G, Le May M, Borentain M, Gershlick A (2006) Percutaneous coronary intervention after fibrinolysis: a multiple meta-analyses approach according to the type of strategy. J Am Coll Cardiol 48:1326-1335. doi:10.1016/j.jacc.2006. 03.064

35. Wilson K, Gibson N, Willan A, Cook D (2000) Effect of smoking cessation on mortality after myocardial infarction. Meta-analysis of cohort studies. Arch Intern Med 160:939-944. doi:10.1001/ archinte.160.7.939

36. Critchley JA, Capewell S (2003) Mortality risk reduction associated with smoking cessation in patients with coronary heart disease. A systematic review. JAMA 290:86-97. doi:10.1001/jama.290. 1.86 